\title{
The case for thermalization as a contributor to the $[\mathrm{C} \mathrm{II}]$ deficit
}

\author{
Jessica Sutter ${ }^{\circledR},{ }^{\star}$ Daniel A. Dale, ${ }^{1}$ Karin Sandstrom, ${ }^{2}$ J. D. T. Smith, ${ }^{3}$ Alberto Bolatto, ${ }^{4}$ \\ Mederic Boquien, ${ }^{5}$ Daniela Calzetti, ${ }^{6}$ Kevin V. Croxall, ${ }^{7}$ Ilse De Looze, ${ }^{8}$ Maud Galametz, ${ }^{9}$ Brent
} A. Groves, ${ }^{10,11}$ George Helou, ${ }^{12}$ Rodrigo Herrera-Camus, ${ }^{13}$ Leslie K. Hunt ${ }^{\circledR},{ }^{14}$ Robert C. Kennicutt, ${ }^{15,16}$ Eric W. Pelligrini, ${ }^{17}$ Christine Wilson ${ }^{\circledR 18}$ and Mark G. Wolfire ${ }^{4}$

${ }^{1}$ Department of Physics \& Astronomy, University of Wyoming, Laramie WY, 82072, USA

${ }^{2}$ Center for Astrophysics and Space Sciences, Department of Physics, University of California, San Diego, CA, 92093, USA

${ }^{3}$ Department of Physics and Astronomy, University of Toledo, Toledo, OH, 43606, USA

${ }^{4}$ Department of Astronomy, University of Maryland, College Park, MD, 20742, USA

${ }^{5}$ Centro de Astronomía (CITEVA), Universidad de Antofagasta, Avenida Angamos 601, Antofagasta, 1270300, Chile

${ }^{6}$ Department of Astronomy, University of Massachusetts, Amherst, MA, 01003, USA

${ }^{7}$ Expeed Software, Columbus, OH, 43085, USA

${ }^{8}$ Department of Physics and Astronomy, University College London, London, WC1E 6BT, UK

${ }^{9}$ Laboratoire AIM, CEA, Université Paris Diderot, IRFU/Service d'Astrophysique, Gif-sur-Yvette, 91191, France

${ }^{10}$ Research School of Astronomy \& Astrophysics, Australian National University, Canberra, ACT 2611, Australia

${ }^{11}$ University of Western Australia, Perth, WA 6009, Australia

${ }^{12}$ IPAC, California Institute of Technology, Pasadena, CA, 91125, USA

${ }^{13}$ Departmento de Astronomía, Facultad de Ciencias Físicas y Matemáticas, Universidad de Concepción, $5 X C 8+68$ Concepción, Chile

${ }^{14}$ INAF - Osservatorio Astrofisico di Arcetri, Firenze, 50125, Italy

${ }^{15}$ Steward Observatory, University of Arizona, Tucson, AZ, 85719, USA

${ }^{16}$ George P. and Cynthia W. Mitchell Institute for Fundamental Physics and Astronomy, Texas A\&M University, College Station, TX, 77843, USA

${ }^{17}$ Institute for Theoretical Astrophysics Heidelberg, 69120 Heidelberg, Germany

${ }^{18}$ Department of Physics and Astronomy, McMaster University, Hamilton, ON, L8S 4L8, Canada

Accepted 2021 February 16. Received 2021 February 1; in original form 2020 December 9

\begin{abstract}
The [C II] deficit, which describes the observed decrease in the ratio of [C II] $158 \mu \mathrm{m}$ emission to continuum infrared emission in galaxies with high star formation surface densities, places a significant challenge to the interpretation of [C II] detections from across the observable universe. In an attempt to further decode the cause of the $[\mathrm{C}$ II] deficit, the $[\mathrm{C}$ II] and dust continuum emission from 18 Local Volume galaxies has been split based on conditions within the interstellar medium where it originated. This is completed using the Key Insights in Nearby Galaxies: a Far-Infrared Survey with Herschel (KINGFISH) and Beyond the Peak (BtP) surveys and the wide-range of wavelength information, from UV to far-infrared emission lines, available for a selection of star-forming regions within these samples. By comparing these subdivided [C II] emissions to isolated infrared emission and other properties, we find that the thermalization (collisional de-excitation) of the [C II] line in $\mathrm{H}$ II regions plays a significant role in the deficit observed in our sample.
\end{abstract}

Key words: (ISM:) H II Regions -(ISM:) photodissociation regions - galaxies: ISM.

\section{INTRODUCTION}

The [C II] $158 \mu \mathrm{m}$ line is an increasingly alluring tool for understanding how galaxies evolve over time. As one of the major cooling channels for the interstellar medium (ISM; Wolfire et al. 2003), [C II] $158 \mu \mathrm{m}$ emission can represent up to a few percent of the total infrared output of a star-forming galaxy (Malhotra et al. 2001; Smith et al. 2017). The far-infrared wavelength of the [C II] line makes it a frequent target of high-redshift surveys, as it falls within the observable bands of millimetre arrays across a wide range of redshifts $(2<z<7)$ (e.g. Rybak et al. 2019; Ginolfi et al. 2020).

^E-mail: jsutter4@uwyo.edu
Except in extreme cases, the long wavelength of the [C II] line also allows it to pass through gas and dust with little to no attenuation, which, along with [C II] line's role as a major ISM coolant, frequently make it the brightest observed emission line in star-forming galaxies (Luhman et al. 2003).

In order to interpret the multitude of new high- $z$, [C II] detections from millimetre observatories like The Atacama Large Millimeter Array (ALMA; e.g Schaerer et al. 2020), it is important that we have a thorough understanding of the production of the [C II] line in well-studied, local volume galaxies. Previous works have suggested that $[\mathrm{C}$ II] could trace star formation rate (SFR; De Looze et al. 2011, 2014; Herrera-Camus et al. 2015), shock-heated gas (Appleton et al. 2017), or diagnose properties of the atomic interstellar medium (Contursi et al. 2002; Herrera-Camus et al. 2017). Before these 
potential uses of the $[\mathrm{CII}]$ line can be applied to high- $z$ [C II] measurements, the limitations of the [C II] line must be understood.

A complication discovered in this pursuit is the [C II] deficit. The $[\mathrm{C} \mathrm{II}]$ deficit describes the decreasing trend in the ratio of [C II] $158 \mu \mathrm{m}$ luminosity to the total infrared luminosity (TIR) from 5 to $1100 \mu \mathrm{m}$ with increasing star formation rates or hotter dust (Malhotra et al. 2001; Smith et al. 2007; Sargsyan et al. 2012; Sutter et al. 2019). This effect has been measured in a wide variety of galaxies, including Ultra-Luminous Infrared Galaxies (ULIRGs; Díaz-Santos et al. 2017; Herrera-Camus et al. 2018) and high-z galaxies (Capak et al. 2015; Decarli et al. 2018). The [C II] deficit is especially detrimental when using the [C II] line as a measure of SFR, since the TIR luminosity, which indicates absorbed UV/optical emission from young stars re-radiated by dust, is expected to be an accurate SFR tracer as well (De Looze et al. 2014; Herrera-Camus et al. 2015).

Multiple processes have been proposed to explain the cause of the [C II] deficit. These include (1) [C II] self-absorption or [C II] becoming optically thick in high-density environments (Abel et al. 2007; Neri et al. 2014), (2) changes in photoelectric heating efficiency caused by the charging of polycyclic aromatic hydrocarbons (PAHs; Malhotra et al. 2001; Croxall et al. 2012), (3) the conversion of singly ionized carbon to doubly ionized carbon in AGN-host galaxies (Langer \& Pineda 2015), (4) thermalization of the [C II] line in highdensity H II regions (Díaz-Santos et al. 2017), (5) [O I] and other far-infrared fine structure lines overcoming [C II] as the dominant cooling sources (Brauher, Dale \& Helou 2008), (6) an increased ionization parameter in dense galaxies leading to a greater fraction of FUV radiation being absorbed by dust and therefore less availability of carbon-ionizing radiation (De Looze et al. 2014; Herrera-Camus et al. 2018), (7) increasing $G_{0} / n$ values within a single PDR are expected to decrease [C II]/TIR values through grain charging and [O I] becoming the dominant cooling line (Kaufman et al. 1999), or some combination of these effects. Determining the precise cause of the [CII] deficit will help establish the utility of measurements of this line in the high-redshift universe.

To study the behaviour of the [C II] deficit, it is important to consider the different environments in which $\mathrm{C}^{+}$can be found. As carbon has a relatively low ionization potential of $11.3 \mathrm{eV}(110 \mathrm{~nm})$, lower than the $13.6 \mathrm{eV}$ necessary to ionize hydrogen, $\mathrm{C}^{+}$is present in many ISM phases, complicating the determination of the cause of the deficit.

This paper builds on the results of Sutter et al. (2019), which measured the $[\mathrm{CII}]$ deficit in a sample of nearby, star-forming galaxies and found the deficit has a more significant effect in the ionized phases of the ISM (i.e. phases where hydrogen is ionized) than in neutral phases. This was found by measuring the clear differences in the trends observed in the $[\mathrm{C}$ II]/TIR values from the isolated ionized and neutral phases as a function of dust temperature (see fig. 5 of Sutter et al. 2019). In order to elucidate the cause of this difference, we present the results from a novel analysis of the [C II] deficit. Employing the wide range of available photometric observations and spectral emission-line detections for this sample, we are able to separate many of the observed fluxes of these galaxies into the phases from which they arose. By isolating emission from separated phases of the ISM, we are able to isolate the effects of potential causes of the $[\mathrm{C} \mathrm{II}]$ deficit. Preliminary results from this analysis suggest that the thermalization of the [C II] line in $\mathrm{HII}$ regions may play a significant role in the [C II] deficit across our sample.

Thermalization occurs when the critical density of a collisional interaction is surpassed. Below the critical density, line intensities increase with increasing density, $I($ line $) \propto n^{2}$, as higher densities lead to a higher frequency of collisional excitations. Above the critical density, or the density at which collisional de-excitations and spontaneous radiative decays are equal, line intensities will linearly increase with increasing density $(I($ line $) \propto n)$ as local thermodynamic equilibrium conditions are approached. It is important to consider thermalization when studying the [C II] deficit as when SFR and thus TIR luminosity increase, the thermal pressure increases leading to an increased density (Ostriker, McKee \& Leroy 2010; Herrera-Camus et al. 2017), while the [C II] line would increase comparatively more slowly. By comparing the density in isolated ISM phases to the [C II]/TIR ratio for these phases, this theoretical cause of the [C II] deficit can be observationally tested.

This paper is organized as follows. Section 2 describes the sample and observations used to complete this analysis. Section 3 explains the methods applied to determine different properties and measurements separated by ISM phase. Finally, Section 5 lays out the initial conclusions obtained in this work.

\section{DATA AND SAMPLE}

This work uses the subset of the Key Insights in Nearby Galaxies: a Far Infrared Survey with Herschel, or KINGFISH, sample with [C II] $158 \mu \mathrm{m},[\mathrm{N}$ II] $122 \mu \mathrm{m}$ and $205 \mu \mathrm{m}$, and [O I] $63 \mu \mathrm{m}$ line detections. The KINGFISH survey includes 61 local volume galaxies with imaging and spectroscopy across the infrared spectrum (Kennicutt et al. 2011). Of the 61 total galaxies, star-forming regions in 18 local galaxies $(D<20 \mathrm{Mpc})$ have the full suite of line observations required for this work. All line measurements were performed using the Photoconductor Array Camera and Spectrometer (PACS) on the Herschel Space Telescope (Poglitsch et al. 2010). 12 of these galaxies were also included in the Beyond the Peak (BtP) survey. This survey used the Spectral and Photometric Imaging Receiver (SPIRE; Griffin et al. 2010) to obtain maps of the [N II] $205 \mu \mathrm{m}$ over a wider field of view than the KINGFISH observations, extending the coverage of these galaxies to the more quiescent areas surrounding the starforming regions targeted at $205 \mu \mathrm{m}$ by the PACS instrument. The combination of the KINGFISH and BtP surveys cover a total of 120 individual regions in these 18 galaxies. All line maps were smoothed to the 14.5 arcsec point spread function (PSF) of the [N II] $205 \mu \mathrm{m}$ maps using a Gaussian profile. For the regions in this sample, the 14.5 arcsec radius translates to a physical length of $200-2100 \mathrm{pc}$. The regions in these two samples have SFR $\sim 0.082-3.92 \mathrm{M}_{\odot} \mathrm{yr}^{-1}$, stellar masses of $\log _{10}\left(\mathrm{M}_{\odot}\right) \sim 8.97-10.84$ and cover $\log _{10}(\mathrm{O} / \mathrm{H})+12$ $\sim 8.1-8.7$, making them all normal, star-forming galaxies (Kennicutt et al. 2011; Hunt et al. 2019). Further information about the data processing and this sample can be found in Sutter et al. (2019).

In addition to the far-infrared line maps, the KINGFISH survey imaged each galaxy at 70, 100, and $160 \mu \mathrm{m}$ using the PACS instrument and at $250 \mu \mathrm{m}$ using the SPIRE instrument on-board Herschel (the KINGFISH 350 and $500 \mu \mathrm{m}$ data are not utilized in this work). All 18 galaxies in this study were also included in the Spitzer Infrared Nearby Galaxies Survey (SINGS; Kennicutt et al. 2003). As part of this survey, each galaxy was imaged at 3.6, 4.5, 5.6, 8.0, and $24 \mu \mathrm{m}$, using the Infrared Array Camera (IRAC) and the Multiband Imagine Photometer (MIPS) on the Spitzer Space Telescope (Rieke et al. 2004). These infrared bands primarily measure the warm dust and light from old stars in these galaxies. Additionally, ugriz data were obtained from the Sloan Digital Sky Survey (SDSS) for each galaxy in this sample. Foreground stars and background galaxies are removed from the SDSS maps. Further descriptions of the ugriz data 


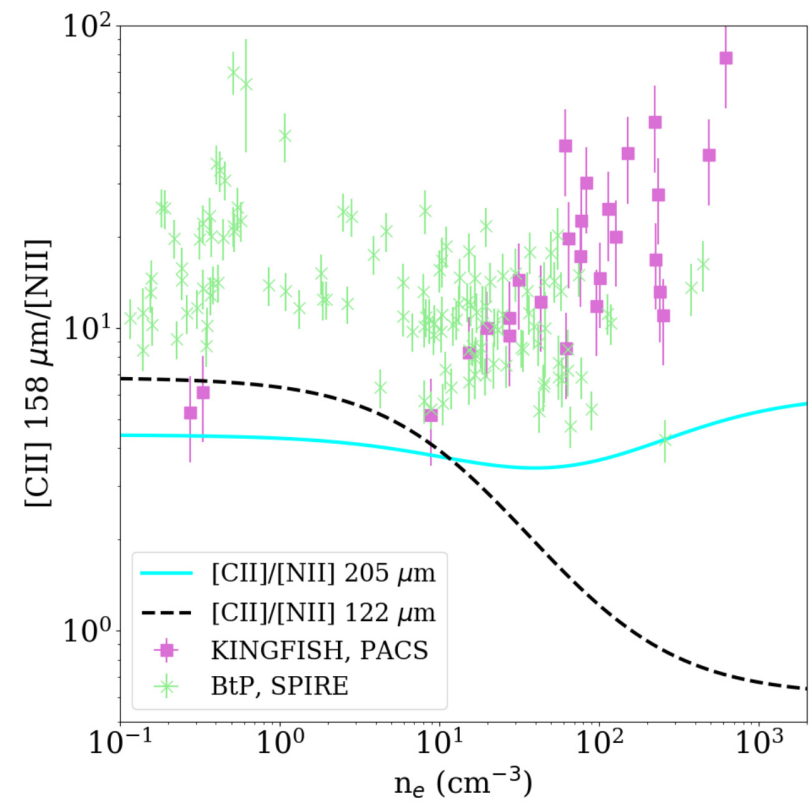

Figure 1. The theoretical ratio of the [C II] $158 \mu \mathrm{m}$ line and [N II] 122 and $205 \mu \mathrm{m}$ lines for ionized gas are represented by the black-dashed line and solid cyan line, respectively. These ratios are used to determine the fraction of the [C II] emission that arises in the ionized phases of the ISM, where both $\mathrm{C}^{+}$ and $\mathrm{N}^{+}$are present. The observed ratio of [C II] $158 \mu \mathrm{m}$ to the [N II] $205 \mu \mathrm{m}$ emission is shown for each region in our sample. As all the observations fall above the cyan line, much of the [C II] emission must originate from the neutral ISM, where no $\mathrm{N}^{+}$is present. Theoretical ratios are determined using the method described in Croxall et al. (2012) and are based on Galactic carbon and nitrogen abundances.

processing can be found in Cook et al. (2014). To expand the range of photometric data, GALEX FUV (1350-1750 ̊) and NUV (1750$2800 \AA$ ) images were also obtained. All galaxies were imaged with GALEX as part of either the Nearby Galaxy Survey (NGS) or the All-Sky Imaging Survey (AIS; Gil de Paz et al. 2005). In order to perform consistent analysis across this wide-range of wavelengths, all imaging data were smoothed to the $250 \mu \mathrm{m}$ PSF of 18 arcsec using a Gaussian profile.

\section{SEPARATION OF IONIZED AND NEUTRAL ISM}

In order to perform this analysis, the ISM within each galaxy was split into two phases. The first phase is the ionized phase, characterized by environments where hydrogen is pre-dominately ionized, and includes $\mathrm{H}$ II regions and the diffuse ionized ISM. The second phase is the neutral phase, where hydrogen is pre-dominately neutral, and includes photodissociation regions (PDRs) as well as molecular clouds. This work proposes a novel method to examine the [C II] deficit by separating not only the [C II] emission but also the TIR luminosity and gas properties by ISM phase in unresolved starforming regions. By isolating the two phases, the precise causes of the deficit can be tested with fewer complicating factors.

\subsection{Isolating the ionized versus neutral [C II] emission}

The [C II] emission was divided by ionized and neutral ISM phases, using the theoretical relationship between the [C II] $158 \mu \mathrm{m}$ line and the $[\mathrm{N} \mathrm{II}] 205 \mu \mathrm{m}$ line in ionized gas. Fig. 1 shows the predicted ratio of the [C II] $158 \mu \mathrm{m}$ line to [N II] emission from the ionized ISM ([C II] $/ 122 \mu \mathrm{m}$ as the black-dashed line, $[\mathrm{C} \mathrm{II}] / 205 \mu \mathrm{m}$ as the solid cyan line) as function of electron number density $\left(n_{\mathrm{e}}\right)$. While the solid cyan line stays at an approximately constant value of 4 , the blackdashed line varies by nearly an order of magnitude across typical ISM density conditions. The measured ratios of [C II] $158 \mu \mathrm{m}$ to the [N II] $205 \mu \mathrm{m}$ emission for the KINGFISH and BtP sample are shown as magenta squares and green crosses, respectively. As nitrogen has an ionization potential of $14.5 \mathrm{eV}$, well above the $13.6 \mathrm{eV}$ necessary to ionize hydrogen, $\mathrm{N}^{+}$should primarily exist in the ionized phases of the ISM, so the theoretical ratio of the $[\mathrm{C} \mathrm{II}] /[\mathrm{N} \mathrm{II}]$ lines can therefore be used to predict the amount of [C II] emission from ionized phases of the ISM. The $205 \mu \mathrm{m}$ line is preferred here over the $122 \mu \mathrm{m}$ line as the critical densities for collisions with electrons for the $205 \mu \mathrm{m}$ line and the $\left[\mathrm{C}\right.$ II] $158 \mu \mathrm{m}$ line are fairly similar $\left(\approx 32 \mathrm{~cm}^{-3}\right.$ for the $[\mathrm{N} \mathrm{II}] 205 \mu \mathrm{m}$ line and $\approx 45 \mathrm{~cm}^{-3}$ for the [C II] $158 \mu \mathrm{m}$ line Oberst et al. 2006; Croxall et al. 2017). This consistency makes the predicted ratio of the 158 and $205 \mu \mathrm{m}$ lines from ionized gas nearly independent of electron number density. In Fig. 1, $n_{\mathrm{e}}$ was determined based on the ratio of the [N II] $122 \mu \mathrm{m}$ and [N II] $205 \mu \mathrm{m}$ lines, as described in Section 3.3.

As all points lie either above or on the predicted ratio for these two emission lines in ionized gas, we know a large amount of the [C II] emission for our sample must be from the neutral phases of the ISM, where no ionized nitrogen is present. We calculate the fraction of the [C II] emission from the neutral ISM using the equation:

$f_{[\mathrm{C} \text { II }] \text {,Neutral }}=\frac{[\mathrm{C} \text { II }] 158-\left(\mathrm{R}_{\text {Ionized }} \times[\mathrm{N} \text { II }] 205\right)}{[\mathrm{C} \text { II }] 158}$,

where $R_{\text {Ionized }}$ is the model ratio of the [C II] $158 \mu \mathrm{m}$ to [N II] $205 \mu \mathrm{m}$ emission from the phases of the ISM where both $\mathrm{C}^{+}$and $\mathrm{N}^{+}$are present and was determined individually for each of the 120 regions using the measured $n_{\mathrm{e}}$ (cf. Croxall et al. 2012). For this work, $R_{\text {Ionized }}$ uses an assumption of a Galactic $\mathrm{C} / \mathrm{N}$ ratio. It is possible that changes in the $\mathrm{C} / \mathrm{N}$ ratio across our sample could have some effects on the measurements of $f_{[\mathrm{C} \mathrm{II}, \text { Neutral }}$, but as shown in Croxall et al. (2017), it is likely that these effects are small compared to variations across the regions in this sample. The method produces measurements of $f_{[\mathrm{C} \mathrm{II}] \text {,Neutral }}$ spanning a range from 2 to 94 per cent with a median value of 66 per cent (see fig. 3 of Sutter et al. 2019), similar to what has been found in other works (Croxall et al. 2012; Parkin et al. 2013; Hughes et al. 2015; Díaz-Santos et al. 2017).

\subsection{Isolating the ionized versus neutral L(TIR)}

The TIR luminosity was determined using the results of Dale et al. (2014):

$$
\begin{aligned}
L(\mathrm{TIR})= & 1.548 v L_{v}(24 \mu \mathrm{m})+0.767 v \mathrm{~L}_{v}(70 \mu \mathrm{m}) \\
& +1.285 v L_{v}(160 \mu \mathrm{m})
\end{aligned}
$$

and represents the integrated luminosity from 5 to $1100 \mu \mathrm{m}$. We then separate the TIR luminosity arising from the ionized versus neutral ISM phases using the results of the spectral energy distribution (SED) fitting of the 3.6-250 $\mu \mathrm{m}$ thermal dust emission constraints. The SED fits were completed using the Code Investigating GALaxy Evolution (CIGALE; Noll et al. 2009; Boquien et al. 2019) and the full range of photometric observations described in Section 2. All SED fits were determined using a Bruzual \& Charlot (2003) stellar population, a modified starburst dust attenuation law, and a Draine et al. (2014) dust emission model. The outputs from the best-fitting model along with the Draine et al. (2007) dust models were applied to estimate the fraction of the TIR luminosity from dust heated within the H II 
regions. These models include a method for predicting the fraction of dust luminosity heated by an interstellar radiation field (ISRF; modelled as $U$ ) above a set cut-off value $U_{c}$. By setting $U_{c}$ to the value of the ISRF expected at the Strömgren radius, or the radius of the $\mathrm{H}$ II region, we can predict the fraction of dust luminosity coming from the ionized HII region surrounding recent star formation. This fraction is determined using equation (18) from Draine et al. (2007):

$$
f\left(L_{\text {dust }} ; U>U_{c}\right)=\frac{\gamma \ln \left(U_{\max } / U_{c}\right)}{(1-\gamma)\left(1-U_{\min } / U_{\max }\right)+\gamma \ln \left(U_{\max } / U_{\min }\right)},
$$

where $\gamma$ is the fraction of the dust luminosity from dust heated by young stars and $U$ is a dimensionless scale factor that when multiplied by the specific energy density of Mathis, Mezger \& Panagia (1983) quantifies the specific energy density of starlight, i.e. the ISRF. $U_{\max }$ is then the maximum value of this scale factor, which is set to $10^{6}$ for each region (as in Draine et al. 2007), $U_{\min }$ is the minimum value of this scale factor, which is determined by the CIGALE SED fitting, and $U_{c}$ is the cut-off value for this scale factor, which is calculated for each region individually using the definition of the Strömgren radius:

$R_{S}=\left(\frac{3 N_{\mathrm{Ly}}}{4 \pi n_{\mathrm{e}}^{2} \alpha}\right)^{1 / 3}$

to determine the expected UV flux within each $\mathrm{H}$ II region. We use an $\alpha=3.0 \times 10^{-13} \mathrm{~cm}^{3} \mathrm{~s}^{-1}$ and estimate $N_{\mathrm{Ly}}$ by integrating the unattenuated UV flux (i.e. the flux between 91.2 and $206.6 \mathrm{~nm}$ ) predicted by the modelled SED. This allows us to estimate ISRF at the Strömgren radius, $U_{c}$, in Habing units $\left(1.6 \times 10^{-3} \mathrm{erg} \mathrm{s}^{-1} \mathrm{~cm}^{-2}\right)$ by dividing the unattenuated UV flux by the surface area of a sphere with the radius determined by equation (4). This gives us a range of $U_{c}$ from $2.0 \times 10^{-1}-1.7 \times 10^{4}$ with a mean value of $7.93 \times 10^{2}$. Using this $U_{c}$ in equation (3), we can determine the fraction of the TIR luminosity from within the $\mathrm{H}$ II region, or $f_{\mathrm{H} \text { II }}$.

For the regions included in this sample, $f_{\mathrm{H}}$ rI ranges from 0.1 to 19 per cent with a median value of 2.6 per cent. The TIR luminosity from the ionized ISM (TIR Ionized $_{\text {) }}$ is $f_{\mathrm{H} \text { II }} \times L$ (TIR), while the neutral TIR luminosity $\left(\right.$ TIR $\left._{\text {Neutral }}\right)$ is the difference between the measured TIR luminosity and $\mathrm{TIR}_{\text {Ionized }}$.

In order to test the viability of this method for isolating the TIR luminosity, measurements of $\mathrm{TIR}_{\text {Ionized }}$ for the subset of regions included in Murphy et al. (2018) are plotted against $L(33 \mathrm{GHz})$ in Fig. 2. The $33 \mathrm{GHz}$ luminosities were corrected to include only the thermal component using the results of Linden et al. (2020). As the thermal component of the $33 \mathrm{GHz}$ luminosity traces free-free emission associated with H II regions (Condon \& Yin 1990; Murphy et al. 2011), it should be well correlated with the TIR emission from $\mathrm{H}$ II regions. As shown in Fig. 2, there is a clear trend between these two properties, with scatter coming mainly from the BtP regions tracing the more quiescent areas surrounding $\mathrm{H}$ II regions. This suggests that our method for determining TIR Ionized $_{\text {works well }}$ for star-forming regions, but there may be added uncertainty for the diffuse ISM.

\subsection{Density measurements for the ionized and neutral ISM}

The densities of the neutral and ionized ISM phases were determined separately for each region in this sample. First, to determine the electron number density within the ionized phases, the ratio of the [N II] 122 and $205 \mu \mathrm{m}$ lines was used (Herrera-Camus et al. 2016). As nitrogen has an ionization potential above that of hydrogen,

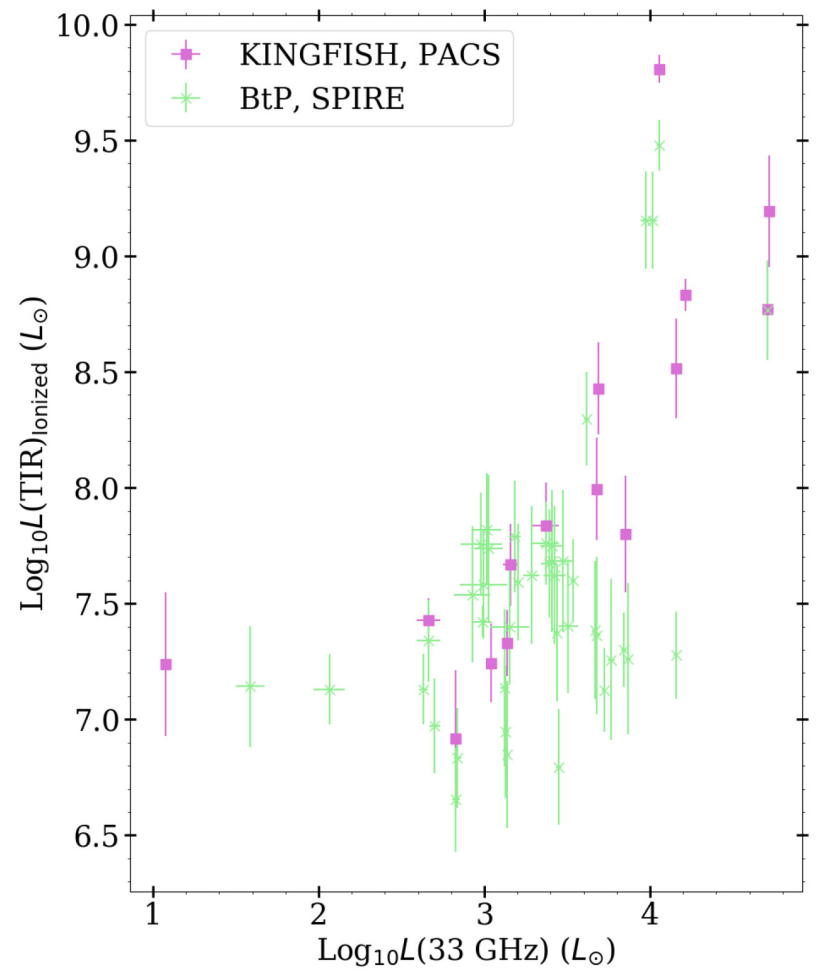

Figure 2. Isolated ionized-phase TIR luminosities plotted against the $33 \mathrm{GHz}$ luminosities measured by Murphy et al. (2018) and corrected to include only the thermal fraction of the radio component using the results of Linden et al. (2020) to demonstrate the applicability of using SED models to separate TIR emission.

both [N II] lines should originate in the ionized phases of the ISM. This ratio has been found to be sensitive to $n_{\mathrm{e}}$ spanning $10-1000 \mathrm{~cm}^{-3}$.

The density of the neutral phases were determined using PDR models. We approximate $[\mathrm{CII}]_{\text {Neutral }}$ emission as originating predominantly from PDRs, where we define PDRs as regions where FUV light dominates the heating and photochemistry within the ISM. Working with this approximation, the PDR models of Kaufman, Wolfire \& Hollenbach (2006) and Pound \& Wolfire (2008) are ideal tools to estimate the properties of the neutral ISM in the regions included in this sample. These models determine the density and FUV radiation intensity $\left(G_{0}\right)$ by comparing the [C II] $158 \mu \mathrm{m}$ and [OI] $63 \mu \mathrm{m}$ line strengths, two of the pre-dominant cooling channels in PDRs, as well as the sum of these two lines, to the total infrared emission, a measurement of the heating in PDRs. The models of hydrogen nucleus density $\left(n_{\mathrm{H}}\right)$ and $G_{0}$ are overplotted on measurements of the [C II] $158 \mu \mathrm{m} /[\mathrm{OI}] 63 \mu \mathrm{m}$ versus the $\left([\mathrm{CII}]_{\text {Neutral }}+[\mathrm{OI}]\right) / \mathrm{TIR}_{\text {Neutral }}$ in Fig. 3 . The $[\mathrm{CII}]$ emission and the TIR luminosity from only the neutral phases were included for this analysis. This method is limited by the effects of self-absorption and attenuation of the shorter wavelength [O I] $63 \mu \mathrm{m}$ line, adding some inherit uncertainty to the measurements of $n_{\mathrm{H}}$ obtained for the neutral ISM.

Using these PDR models, we find the regions in our sample cover a range of $G_{0}$ from 40 to $10^{4}$, with a median value of 250 and a range of $n_{\mathrm{H}}$ from 20 to $10^{3.5} \mathrm{~cm}^{-3}$ with a median value of 500 . These results are what is expected for typical PDRs and agree well with the work done in Malhotra et al. (2001) and Croxall et al. (2012). 


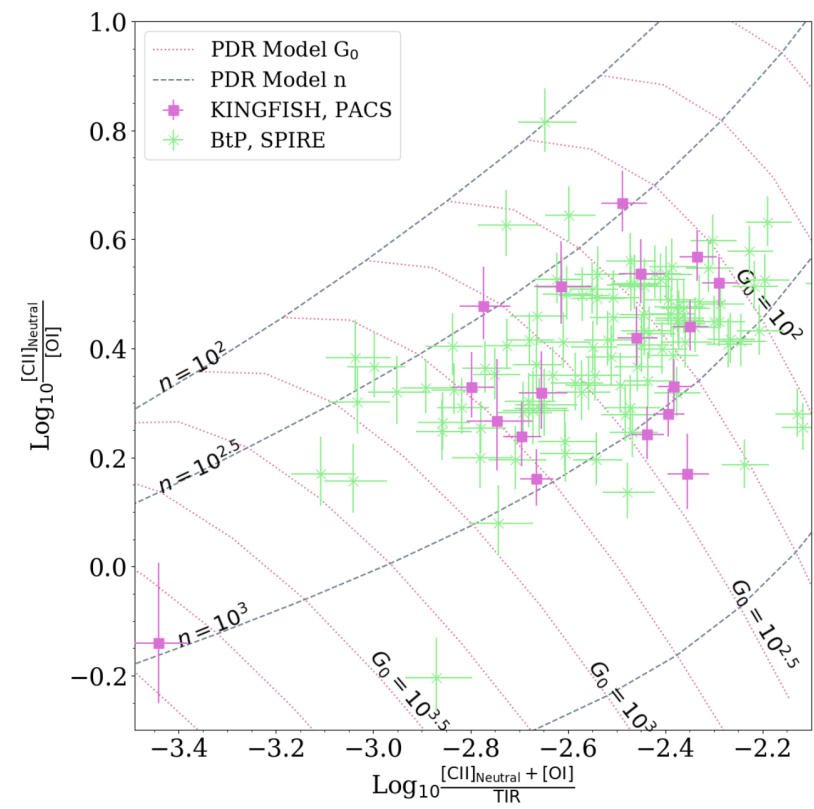

Figure 3. This plot illustrates the PDR models used to determine $n_{\mathrm{H}}$ for the neutral ISM. The ratio of the [C II] emission from the neutral ISM and the [O I] $63 \mu \mathrm{m}$ line emission is plotted against the sum of the luminosity of these two lines divided by the TIR luminosity, a proxy for photoelectric heating efficiency. Overplotted are the $n_{\mathrm{H}}$ and $G_{0}$ measurements determined by the PDR models of Kaufman et al. (2006) and Pound \& Wolfire (2008).

\section{THERMALIZATION AND THE [C II] DEFICIT}

\subsection{Observations of the [C II] deficit}

In order to characterize the $[\mathrm{C}$ II $]$ deficit behaviour observed in this sample, the $[\mathrm{CII}] / \mathrm{TIR}$ measurements are plotted against the $v f_{v}(70 \mu \mathrm{m}) / \nu \mathrm{f}_{v}(160 \mu \mathrm{m})$ values in Fig. 4 . The $v f_{v}(70 \mu \mathrm{m}) / \nu \mathrm{f}_{v}(160 \mu \mathrm{m})$ flux ratio is an indicator of dust temperature, and therefore star formation activity, and is frequently used to show the effects of the [C II] deficit (e.g. Malhotra et al. 1997; Croxall et al. 2012; Díaz-Santos et al. 2013). The left-hand panel of this figure shows the $[\mathrm{C} \mathrm{II}] /$ TIR ratio before any divisions by ISM phase are performed to either the [C II] or TIR luminosities. There is a slight decreasing trend with $\nu f_{v}(70 \mu \mathrm{m}) / \nu \mathrm{f}_{\nu}(160 \mu \mathrm{m})$, indicating the regions in this sample experience moderate [C II] deficit behaviour. The middle panel of Fig. 4 displays the normalized isolated ionized phase $[\mathrm{CII}] / \mathrm{TIR}$ measurements and the right-hand panel shows the normalized isolated neutral phase [C II]/TIR measurements. All data have been normalized by dividing each point by the average value of $[\mathrm{CII}] / \mathrm{TIR}$ for that phase. There is a clear decreasing trend between $v f_{v}(70 \mu \mathrm{m}) / \nu \mathrm{f}_{v}(160 \mu \mathrm{m})$ and $\left([\mathrm{C} \text { II]/TIR })_{\text {Ionized. This }}\right.$ observed decreasing trend in the ionized phases of the ISM, along with the lack of a trend measured in the neutral phases (as shown in the right-hand panel of Fig. 4) suggest that the cause of the slight decreasing trend for the star-forming regions included in this study is occurring in the ionized phases of the ISM. For this reason, we focus our search for causes of the [C II] deficit on trends we measure in the isolated ionized ISM phase [C II] and TIR luminosities.

\subsection{Indications of thermalization}

One finding from this novel approach of tracking the [C II] deficit across the isolated ISM phases is the strong dependence of the observed deficit in the ionized phases of the ISM on the electron density relative to the critical density for the [C II] line. This is shown in Fig. 5, where the [C II]/TIR ratios for the isolated ionized and neutral ISM components are plotted against the electron number density for the ionized ISM, where free electrons are the primary source of collisional excitation of the [C II] line, and the hydrogen nucleus number density for the neutral ISM. Hydrogen atoms are the primary source of collisional excitation of the [C II] line at low column densities and molecular hydrogen dominates at the higher column densities (e.g. Herrera-Camus et al. 2015). The vertical dashed and dotted lines represent the critical electron and hydrogen atom number densities of [C II], respectively (Goldsmith et al. 2012). These critical densities are determined assuming a temperature of $8000 \mathrm{~K}$ for the ionized phase and $100 \mathrm{~K}$ for the neutral phase. The critical density for collisions with molecular hydrogen is a factor of 1.4 higher than for collisions with hydrogen atoms (Wiesenfeld \& Goldsmith 2014). We see that in the ionized phases of the ISM, the [C II]/TIR ratio plummets when the electron number density reaches the critical density. This suggests that the thermalization of the [C II] line is a factor in the observed [C II] deficit in the ionized phases of the ISM. In regions with densities above the critical density, increased heating of the dust and gas from young stars will increase the TIR luminosity, without increasing the [C II] emission strength.

In the left-hand panel of Fig. 5, the sharp decrease along $n_{\text {crit }}$ is primarily observed in the KINGFISH regions, represented by the pink squares. This is likely a reflection of the selection of bright infrared regions for the KINGFISH observations. Alternatively, the wider field of view maps obtained by the BtP survey cover the more quiescent areas within the ISM, where thermalization of the [C II] is not occurring. Although the KINGFISH regions cover areas of more intense star formation, none of the regions included in this sample probes the most extreme conditions where the largest deficits have been observed, as the BtP and KINGFISH sample cover a modest range of $L$ (TIR) of $1 \times 10^{8}-7 \times 10^{11} \mathrm{~L}_{\odot}$.

Despite an overall clear match between the theoretical values and observed data, there are a small subset of BtP regions below $n_{\text {crit }}$ that have lower than predicted [C II]/TIR (see green crosses in the lower right on the left-hand panel of Fig. 5) as well as two BtP regions above $n_{\text {crit }}$ that are higher than expected (see green crosses in the upper right on the left-hand panel of Fig. 5). As all the BtP regions that fall far below the theoretical predictions have larger than average $f_{\mathrm{H} \mathrm{II}}$ values, errors in how $f_{\mathrm{H} \text { II }}$ is determined are likely driving this discrepancy for some of these more quiescent BtP regions. On the other hand, the points above the critical density and theoretical predictions have higher fractions of [C II] emission from the ionized phases, which drives the ([C II]/TIR) Ionized values higher. This could again be caused by a difference in the observed region and the assumptions made by modelling each region as an ionized $\mathrm{H}$ II region surrounded by a neutral PDR.

It is important to note that for these samples, the declining [C II] / TIR values as a function of density only occurs in the ionized ISM. However, it is possible that the measurements of $([\mathrm{C} \text { II }] / T I R)_{\text {Neutral }}$ could also be effected by thermalization, but our simplistic models of the neutral medium having a temperature of $100 \mathrm{~K}$ and therefore a critical density of $3000 \mathrm{~cm}^{-3}$ could be obscuring any effect thermalization has on the $([\mathrm{C} \mathrm{II}] / \mathrm{TIR})_{\text {Neutral }}$ measurements.

\subsection{Theoretical predictions of thermalization}

The observations discussed in Section 4.2 suggest that thermalization of the $[\mathrm{C}$ II $] 158 \mu \mathrm{m}$ line is playing a role in the $[\mathrm{C}$ II] deficit observed 

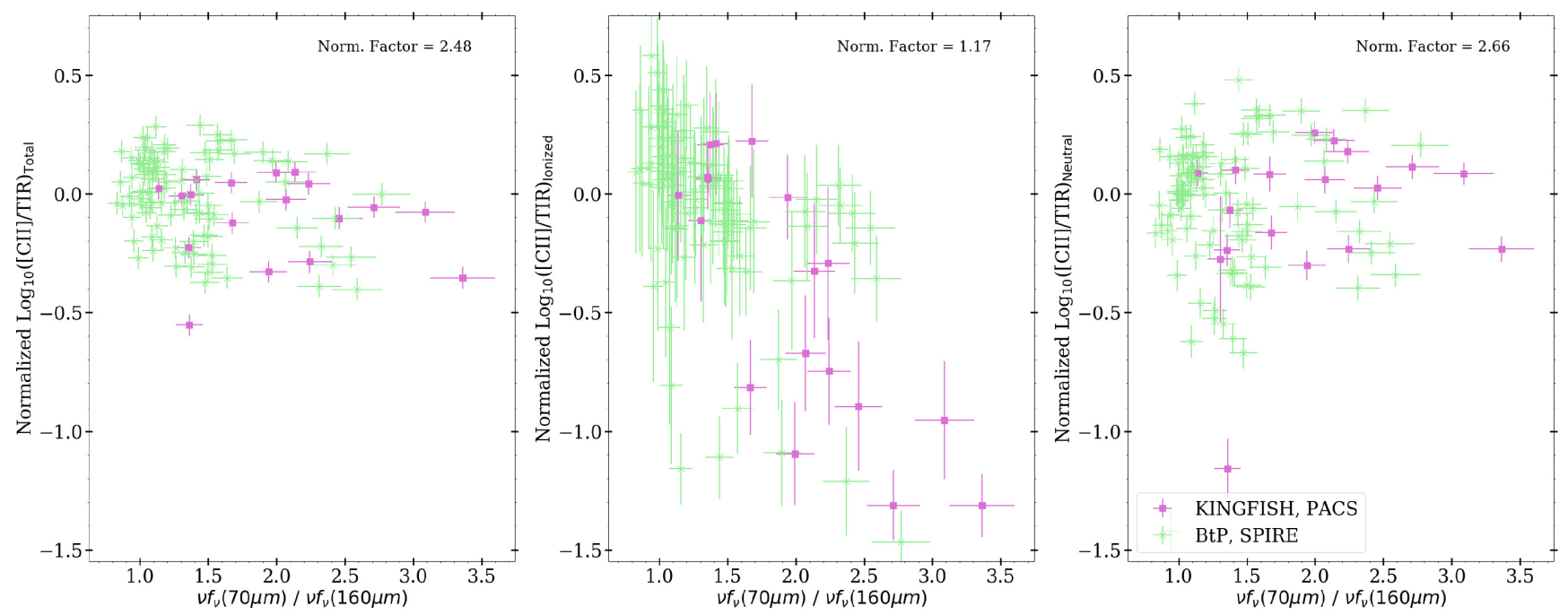

Figure 4. Left: The undivided $[\mathrm{CII}] / \mathrm{TIR}$ measurements are plotted against $v f_{v}(70 \mu \mathrm{m}) / v \mathrm{f}_{v}(160 \mu \mathrm{m})$ flux ratios to show the moderate deficit behaviour observed in the galaxies in this sample. Middle: The isolated ionized phase [C II]/TIR measurements plotted against $\nu f_{\nu}(70 \mu \mathrm{m}) / \nu \mathrm{f}_{\nu}(160 \mu \mathrm{m})$. Notice the steeper negative slope indicating the strong deficit behaviour in the ionized phase. Right: The isolated neutral phase [C II]/TIR measurements plotted against $v f_{v}(70 \mu \mathrm{m}) / \nu f_{v}(160 \mu \mathrm{m})$. No deficit behaviour is observed when only the neutral phase is considered. This builds on the work performed in Sutter et al. (2019). All [C II]/TIR ratios have been normalized by adding the absolute value of the average $\log _{10}[\mathrm{C}$ II]/TIR ratio for the given phase to highlight the different trends in each phase. For clarity, the normalization factors are seen at the top of each panel.
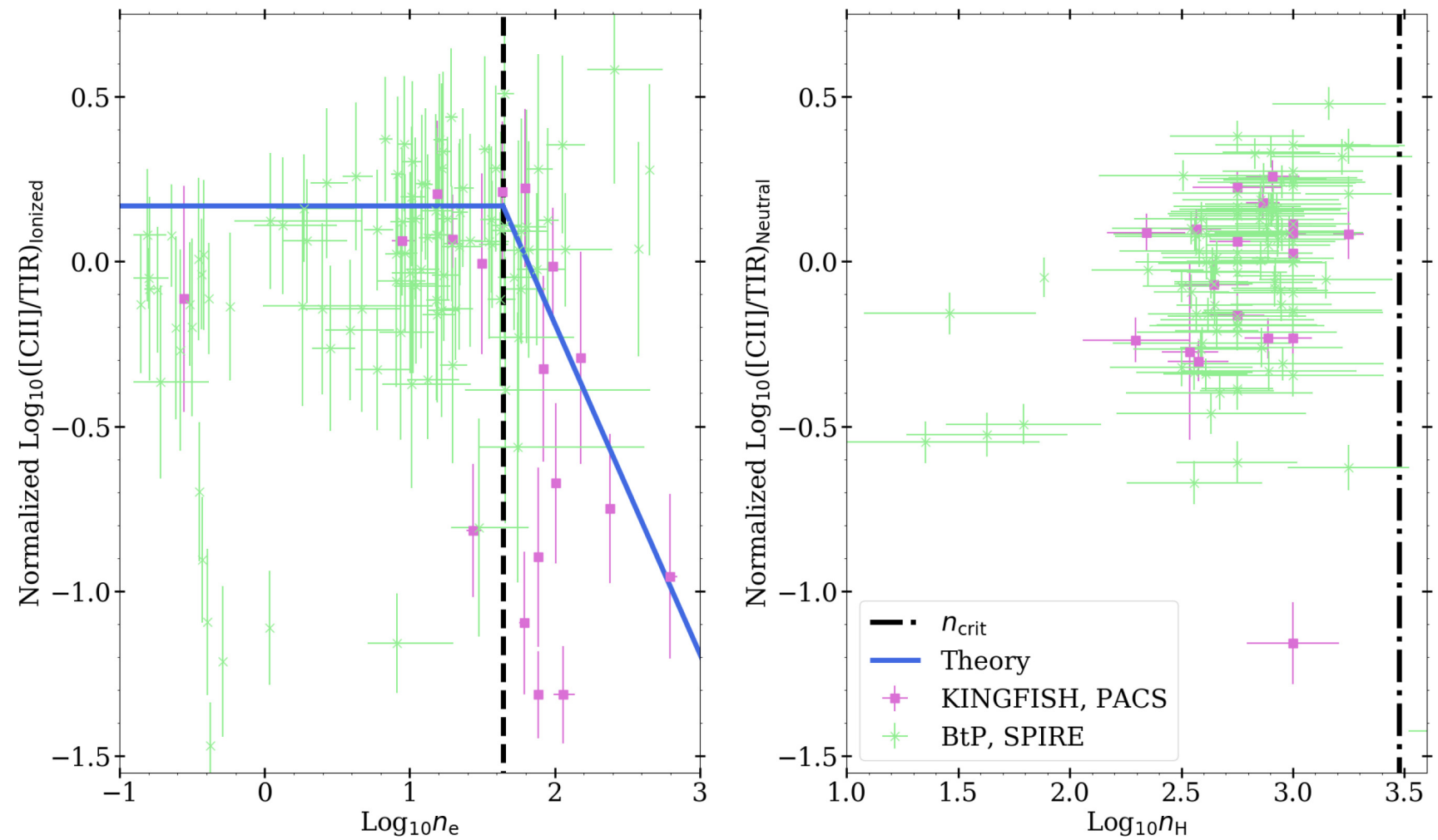

Figure 5. The isolated-ISM phase ratios of [C II]/TIR luminosity for the ionized (left) and neutral (right) phases of the ISM plotted against the electron number density in the ionized phase and the hydrogen atom number density for the neutral phase. The differing number densities were used as electrons are the primary source of collisional excitation for the [C II] line in the ionized phases of the ISM, whereas hydrogen atoms are the primary source of collisional excitations of the $[\mathrm{C} \mathrm{II}]$ line in PDRs. The black dotted and dashed lines represent the critical density of the $[\mathrm{C} \mathrm{II}]$ line for collisions with electrons $\left(50 \mathrm{~cm}^{-3}\right)$ and hydrogen atoms $\left(3 \times 10^{3} \mathrm{~cm}^{-3}\right)$, respectively (Malhotra et al. 2001). The critical density for molecular hydrogen is a factor of 1.4 higher. The solid line in the left-hand panel represents the median value of $\log _{10}([\mathrm{C} \text { II }] / T I R)_{\text {Ionized }}$ for regions with densities below the critical density. The same normalization used in Fig. 4 is applied here. 
in the ionized ISM. This conclusion is supported by a theoretically derived model of the expected TIR and [C II] luminosities, as elucidated in the following discussion. Below the critical density, the [C II] $158 \mu \mathrm{m}$ luminosity from $\mathrm{H}$ II regions should be equal to the number of collisional excitations of $\mathrm{C}^{+}$multiplied by the energy produced by each transition:

$L\left([\mathrm{C}\right.$ II $\left.], n<n_{\text {crit }}\right)=\frac{4}{3} \pi R^{3} n_{e} n_{\mathrm{C}^{+}} \gamma_{[\mathrm{C} \mathrm{II}]} E_{158}$,

where $\gamma_{[\mathrm{CI}]}$ is the rate coefficient for collisional excitations, $R$ is the radius of the $\mathrm{H}$ II region, $E_{158}$ is the energy of a single transition, and $n_{\mathrm{e}}$ and $n_{\mathrm{C}^{+}}$are the electron and singly ionized carbon densities, respectively. Above the critical density, the [C II] luminosity will no longer depend on $n_{e}$, and can therefore be modelled as

$L\left([\mathrm{C} \mathrm{III}], n>n_{\text {crit }}\right)=\frac{4}{3} \pi R^{3} n_{[\mathrm{C} \mathrm{II}]} \gamma_{[\mathrm{C} \text { II }]} E_{158}$.

Regardless of density, the TIR luminosity from $\mathrm{H}$ II regions can be modelled as

$L(\mathrm{TIR})=N_{\mathrm{Ly}} E_{\mathrm{UV}} f_{\mathrm{IR}}$,

where $N_{\mathrm{Ly}}$ is the number of Lyman continuum photons, $E_{\mathrm{UV}}$ is the average energy of the Lyman continuum photons, and $f_{\mathrm{IR}}$ is the fraction of energy from the Lyman continuum photons that is converted to dust heating. Using Strömgren conditions, $N_{\mathrm{Ly}}$ is

$N_{\mathrm{Ly}}=\frac{4}{3} \pi R^{3} n_{e}^{2} \alpha$,

where $\alpha$ is the recombination rate coefficient, and is approximately equal to $3 \times 10^{-13} \mathrm{~cm}^{3} \mathrm{~s}^{-1}$. Using these predictions for the $[\mathrm{C} \mathrm{II}]$ and TIR luminosity for the ionized phase of the ISM, we can estimate the [C II]/TIR ratio as a function of $n_{\mathrm{e}}$. Below the critical density, [C II]/TIR simplifies to

$\frac{[\mathrm{C} \mathrm{II}]}{\mathrm{TIR}}=\frac{n_{[\mathrm{C} \text { II] }} \gamma_{[\mathrm{CH}]} E_{158}}{n_{e} \alpha E_{\mathrm{UV}} f_{\mathrm{IR}}}$.

If we assume $n_{\text {[C II] }} \approx 1 \times 10^{-4} n_{\mathrm{e}}$ based on the Galactic carbon abundances $\left(1.6 \times 10^{-4}\right.$ carbon atoms per hydrogen atom, Sofia et al. 2004), we can further simplify equation (9) to

$\frac{[\mathrm{C} \mathrm{II}]}{\mathrm{TIR}}=\frac{1 \times 10^{-4} \gamma_{[\mathrm{C} \text { II] }} E_{158}}{\alpha E_{\mathrm{UV}} f_{\mathrm{IR}}}$.

Using $\alpha=3 \times 10^{-13} \mathrm{~cm}^{3} \mathrm{~s}^{-1}, \gamma_{[\mathrm{C} \mathrm{II}]}=3.8 \times 10^{-7}$ (Tielens 2005), $E_{\mathrm{UV}}$ of $15 \mathrm{eV}$, and $f_{\mathrm{IR}}=0.5$ as suggested by Inoue (2001), we find

$\frac{[\mathrm{C} \mathrm{II}]}{\mathrm{TIR}}=0.13$

for the ionized phases of the ISM, when $n_{\mathrm{e}} \leq n_{\text {crit }}$. This value is displayed as a blue, horizontal line on the left-hand panel of Fig. 5, and is fairly consistent with the observed $\frac{[\mathrm{CII}]}{\mathrm{TIR}}$ values. As $f_{\mathrm{IR}}=0.5$ is a lower limit for the fraction of UV light reprocessed by dust, it is likely that the slight difference in the theory line and measured values is due to differences in this fraction across our sample (Inoue 2001).

Above the critical density, we expect the [C II] luminosity should no longer be proportional to $n_{\mathrm{e}}$. The predicted $\frac{[\mathrm{CH}]}{\mathrm{TIR}}$ above $n_{\text {crit }}$ is then

$\frac{[\mathrm{C} \mathrm{II}]}{\mathrm{TIR}}=\frac{0.13 n_{\mathrm{crit}}}{n_{e}}$.

The theoretical predictions described in this section are displayed as a solid blue line on the left-hand panel of Fig. 5. It is likely that the theoretical predictions lie below the observed values due to the possibility that some of the $[\mathrm{C}$ II] emission from ionized phases originates in the diffuse ionized ISM, while these predictions only account for the $[\mathrm{C} \mathrm{II}]$ emission from $\mathrm{H}$ II regions. Emission from the diffuse ionized ISM is also expected to play a greater role in the BtP data points, which cover the areas surrounding the starforming regions included in the KINGFISH study. The discrepancy between the theoretical curve and the observed measurements could also be driven by underestimates in our prediction for $f_{\mathrm{IR}}$. As Inoue (2001) focuses on star-forming regions in local galaxies and states $f_{\mathrm{IR}}=0.5$ as an upper limit, it is likely that differences in $A_{V}$ or other conditions in the star-forming regions targeted in this study could lead to variations in the amount of UV light absorbed by dust, increasing the value of $f_{\mathrm{IR}}$.

\section{DISCUSSION AND CONCLUSION}

As previous studies have found that typically $\approx 75$ per cent of [C II] emission originates in PDRs and other neutral phases of the ISM (Rigopoulou et al. 2013; Croxall et al. 2017), thermalization of [C II] in the ionized ISM can only account for moderate overall deficit behaviour. For the normal star-forming galaxies in this work, the undivided $[\mathrm{C}$ II $] /$ TIR measurements showed only moderate deficit behaviour. Therefore, the [C II] deficit observed in this sample can be explained by thermalization of the [C II] line within the ionized phases of the ISM, despite the majority of [C II] emission originating in the neutral ISM (see Fig. 4 or Sutter et al. 2019 for further discussion). Furthermore, by correcting for the effects of thermalization in the ionized phases of the ISM, the deficit effect can be greatly reduced. This is shown by the differences in the lefthand and right-hand panels of Fig. 6. The left-hand panel shows the unmodified [C II]/TIR measurements for the regions in this sample colour coded by $\log _{10} n_{\mathrm{e}}$. The total [C II]/TIR measurements show a slight decline as a function of $\nu f_{v}(70 \mu \mathrm{m}) / \nu \mathrm{f}_{\nu}(160 \mu \mathrm{m})$, a proxy for dust temperature. This decrease is removed in the right-hand panel of Fig. 6, where the [C II]/TIR measurements have been corrected for the effects of thermalization in the ionized ISM. This correction is made using the theoretical predictions described in Section 4.3. For regions above the critical density, the $[\mathrm{C} \mathrm{II}] / \mathrm{TIR}$ values are corrected by adding the correction factor:

$C=0.13\left(1.0-\frac{n_{\text {crit }}}{n_{\mathrm{e}}}\right) \times f_{\mathrm{H} \mathrm{II}}$

Here, $C$ represents the difference between the theoretical expectation of non-thermalized [C II]/TIR $(0.13)$ and thermalized [C II]/TIR $\left(\frac{0.13 n_{\text {crit }}}{n_{\mathrm{e}}}\right)$ from the ionized phases of the ISM. The lefthand panel of Fig. 6 plots $\log _{10}([\mathrm{CII}] / \mathrm{TIR}+\mathrm{C})$ against the $\nu f_{v}(70 \mu \mathrm{m}) / \nu \mathrm{f}_{v}(160 \mu \mathrm{m})$ measurements. This correction for the effects of thermalization removes the observed decrease seen in the undivided $[\mathrm{C}$ II]/TIR data, but still shows a large scatter, indicating that there are likely other physical processes effecting the $[\mathrm{C}$ II]/TIR ratio in these galaxies.

Thermalization may also be the cause for deficits observed in other ionized gas tracers, like [N II] and [O III] lines (Graciá-Carpio et al. 2011; Herrera-Camus et al. 2016), and therefore should be accounted for in studies of ionized gas in galaxies. As the deficit is most prominent in the ionized gas, it is also possible that some of this behaviour is due to increasing levels of doubly ionized carbon. With our current data set, it is difficult to disentangle how thermalization of $[\mathrm{C} \mathrm{II}]$ and a larger fraction of $\mathrm{C}^{++}$both contribute to the observed deficit in the ionized ISM.

It should be noted that despite the evidence presented here, thermalization in the ionized phases of the ISM cannot be the only contributor to the $[\mathrm{C} \mathrm{II}]$ deficit. This is especially true when 

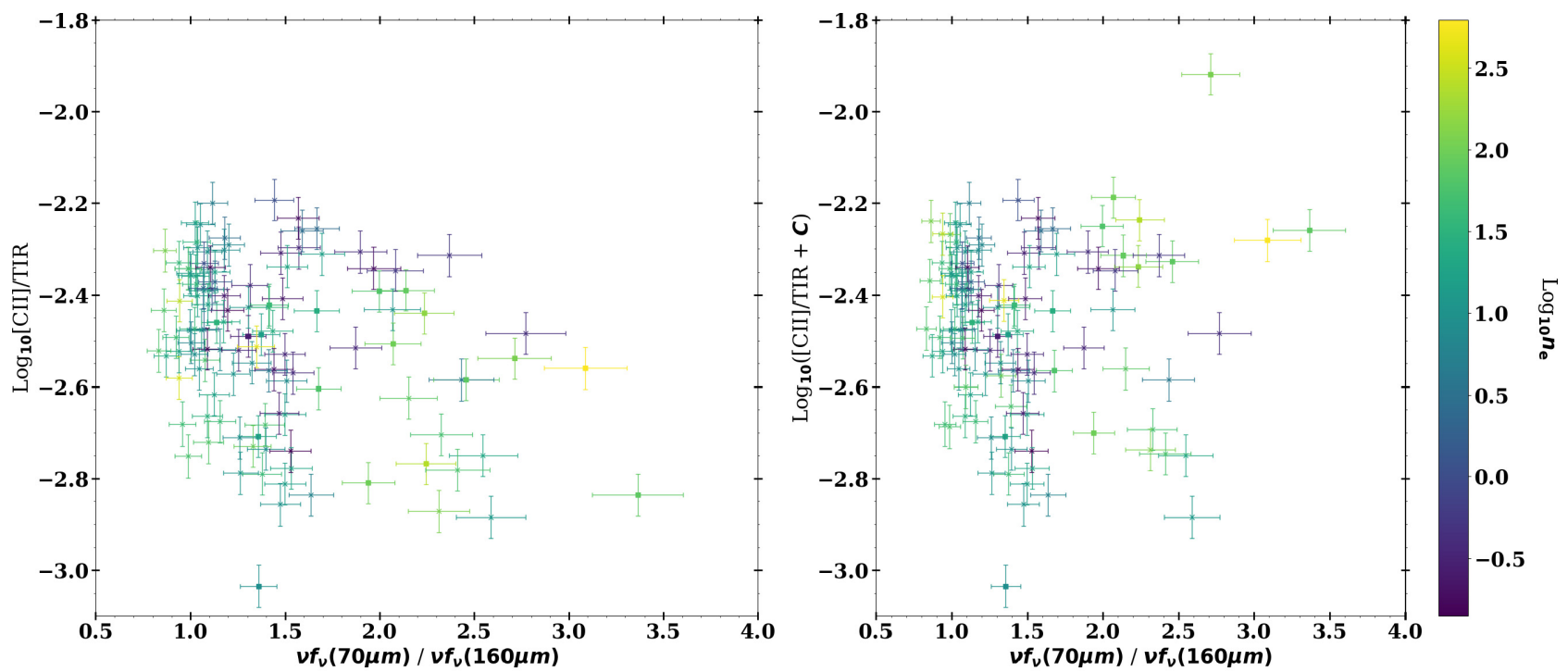

Figure 6. Left-hand panel: The total $[\mathrm{C} \mathrm{II}] / \mathrm{TIR}$ measurements for the regions in this sample colour-coded by $\log _{10} n_{\mathrm{e}}$ against $\nu f_{v}(70 \mu \mathrm{m}) / \nu \mathrm{f}_{v}(160 \mu \mathrm{m})$, an indicator of dust temperature. Filled square points represent KINGFISH regions, while crosses represent the BtP regions. Right-hand panel: Same as the left-hand panel, with the $[\mathrm{C}$ II]/TIR ratios corrected for the effects of thermalization through the addition of the correction factor $C$ described in equation (13), removing the observed deficit.

considering the high- $z$ galaxies with especially pronounced deficit behaviour. For example, the $z \sim 2-6$ galaxies observed in Rawle et al. (2014), Capak et al. (2015), De Breuck et al. (2014), Gallerani et al. (2012), and Malhotra et al. (2017) show [C II]/TIR ratios 10 to 100 times lower than those observed in the local star-forming galaxies included in this work. As the majority of [C II] emission has been found to arise from the neutral phases of the ISM (Pineda et al. 2013; Abdullah et al. 2017; Croxall et al. 2017; Cormier et al. 2019; Sutter et al. 2019; Bigiel et al. 2020), the effects of thermalization solely within the ionized phases of the ISM can only contribute to moderate deficit behaviour. This suggests that although thermalization of the [C II] line within the ionized phases of the ISM may play an important role in the $[\mathrm{C} \mathrm{II}]$ deficit, especially over the kpc-scale star-forming regions included in this work, it cannot be the only factor consider to drive this behaviour across a wide spectrum of spatial resolutions and galaxy conditions.

Although thermalization of [C II] in H II regions cannot completely explain the large deficits observed in some starbursting galaxies (Smith et al. 2017) or the [C II] $158 \mu \mathrm{m}$ emission behaviour in lowmetallicity dwarfs where little [C II] emission originates in ionized phases (Cormier et al. 2015), the sharp decrease in ([C II] / TIR) Ionized along $n_{\text {crit }}$ is an intriguing result that will help us decode the behaviour of the [C II] deficit across the range of galaxies in which it has been observed. Further studies of these isolated ISM phases will help define what other process could affect the strength of the [C II] line, and will have important implications for the use of this line as a tracer of conditions in the high-redshift universe.

\section{ACKNOWLEDGEMENTS}

This work was supported by NASA Headquarters under the NASA Earth and Space Science Fellowship Program, Grant \#80NSSC18K1107, as the Wyoming NASA Space Grant Consortium, NASA Grant \#NNX15AI08H. Herschel is an ESA space observatory with science instruments provided by European-led Principal Investigator consortia and with important participation from NASA. IRAF, the Image Reduction and Analysis Facility, has been developed by the National Optical Astronomy Observatories and the Space Telescope Science Institute.

\section{DATA AVAILABILITY}

All data are available through IRSA through the KINGFISH database at https://irsa.ipac.caltech.edu/data/Herschel/KINGFISH/ with the exception of the SPIRE spectroscopy, which is available upon request.

\section{REFERENCES}

Abdullah A. et al., 2017, ApJ, 842, 4

Abel N. P., Sarma A. P., Troland T. H., Ferland G. J., 2007, ApJ, 662, 1024

Appleton P. N. et al., 2017, ApJ, 836, 76

Bigiel F. et al., 2020, ApJ, 903, 30

Boquien M., Burgarella D., Roehlly Y., Buat V., Ciesla L., Corre D., Inoue A. K., Salas H., 2019, A\&A, 622, A103

Brauher J. R., Dale D. A., Helou G., 2008, ApJS, 178, 280

Bruzual G., Charlot S., 2003, MNRAS, 344, 1000

Capak P. L. et al., 2015, Nature, 522, 455

Condon J. J., Yin Q. F., 1990, ApJ, 357, 97

Contursi A. et al., 2002, AJ, 124, 751

Cook D. O. et al., 2014, MNRAS, 445, 881

Cormier D. et al., 2015, A\&A, 578, A53

Cormier D. et al., 2019, A\&A, 626, A23

Croxall K. V. et al., 2012, ApJ, 747, 81

Croxall K. V. et al., 2017, ApJ, 845, 96

Dale D. A., Helou G., Magdis G. E., Armus L., Díaz-Santos T., Shi Y., 2014, ApJ, 784, 83

De Breuck C. et al., 2014, A\&A, 565, A59

De Looze I., Baes M., Bendo G. J., Cortese L., Fritz J., 2011, MNRAS, 416, 2712

De Looze I. et al., 2014, A\&A, 568, A62

Decarli R. et al., 2018, ApJ, 854, 97

Díaz-Santos T. et al., 2013, ApJ, 774, 68

Díaz-Santos T. et al., 2017, ApJ, 846, 32

Draine B. T. et al., 2007, ApJ, 663, 866

Draine B. T. et al., 2014, ApJ, 780, 172 
Gallerani S. et al., 2012, A\&A, 543, A114

Gil de Paz A. et al., 2005, ApJ, 619, L115

Ginolfi M. et al., 2020, A\&A, 633, A90

Goldsmith P. F., Langer W. D., Pineda J. L., Velusamy T., 2012, ApJS, 203, 13

Graciá-Carpio J. et al., 2011, ApJ, 728, L7

Griffin M. J. et al., 2010, A\&A, 518, L3

Herrera-Camus R. et al., 2015, ApJ, 800, 1

Herrera-Camus R. et al., 2016, ApJ, 826, 175

Herrera-Camus R. et al., 2017, ApJ, 835, 201

Herrera-Camus R. et al., 2018, ApJ, 861, 94

Hughes T. M. et al., 2015, A\&A, 575, A17

Hunt L. K. et al., 2019, A\&A, 621, A51

Inoue A. K., 2001, AJ, 122, 1788

Kaufman M. J., Wolfire M. G., Hollenbach D. J., Luhman M. L., 1999, ApJ, 527,795

Kaufman M. J., Wolfire M. G., Hollenbach D. J., 2006, ApJ, 644, 283

Kennicutt R. C. Jr et al., 2003, Publ. Astron. Soc. Pac., 115, 928

Kennicutt R. C. et al., 2011, PASP, 123, 1347

Langer W. D., Pineda J. L., 2015, A\&A, 580, A5

Linden S. T., Murphy E. J., Dong D., Momjian E., Kennicutt R. C. J., Meier D. S., Schinnerer E., Turner J. L., 2020, ApJS, 248, 25

Luhman M. L., Satyapal S., Fischer J., Wolfire M. G., Sturm E., Dudley C. C., Lutz D., Genzel R., 2003, ApJ, 594, 758

Malhotra S. et al., 1997, ApJ, 491, L27

Malhotra S. et al., 2001, ApJ, 561, 766

Malhotra S. et al., 2017, ApJ, 835, 110

Mathis J. S., Mezger P. G., Panagia N., 1983, A\&A, 500, 259

Murphy E. J. et al., 2011, ApJ, 737, 67

Murphy E. J., Dong D., Momjian E., Linden S., Kennicutt R. C. J., Meier D. S., Schinnerer E., Turner J. L., 2018, ApJS, 234, 24
Neri R., Downes D., Cox P., Walter F., 2014, A\&A, 562, A35

Noll S., Burgarella D., Giovannoli E., Buat V., Marcillac D., Muñoz-Mateos J. C., 2009, A\&A, 507, 1793

Oberst T. E. et al., 2006, ApJ, 652, L125

Ostriker E. C., McKee C. F., Leroy A. K., 2010, ApJ, 721, 975

Parkin T. J. et al., 2013, ApJ, 776, 65

Pineda J. L., Langer W. D., Velusamy T., Goldsmith P. F., 2013, A\&A, 554, A103

Poglitsch A. et al., 2010, A\&A, 518, L2

Pound M. W., Wolfire M. G., 2008, in Argyle R. W., Bunclark P. S., Lewis J. R., eds, ASP Conf. Ser. Vol. 394, Astronomical Data Analysis Software and Systems. Astron. Soc. Pac., San Francisco, p. 654

Rawle T. D. et al., 2014, ApJ, 783, 59

Rieke G. H. et al., 2004, ApJS, 154, 25

Rigopoulou D. et al., 2013, MNRAS, 434, 2051

Rybak M. et al., 2019, ApJ, 876, 112

Sargsyan L. et al., 2012, ApJ, 755, 171

Schaerer D. et al., 2020, A\&A, 643, A3

Smith J. D. T. et al., 2007, ApJ, 656, 770

Smith J. D. T. et al., 2017, ApJ, 834, 5

Sofia U. J., Lauroesch J. T., Meyer D. M., Cartledge S. I. B., 2004, ApJ, 605, 272

Sutter J. et al., 2019, ApJ, 886, 60

Tielens A. G. G. M., 2005, Physics and Chemistry of the Interstellar Medium. Cambridge Univ. Press, Cambridge

Wiesenfeld L., Goldsmith P. F., 2014, ApJ, 780, 183

Wolfire M. G., McKee C. F., Hollenbach D., Tielens A. G. G. M., 2003, ApJ, 587,278

This paper has been typeset from a $\mathrm{T}_{\mathrm{E}} \mathrm{X} / \mathrm{L} \mathrm{T} \mathrm{E} \mathrm{X}$ file prepared by the author. 